\section{REINNERVATION OF RAT EXTENSOR DIGITORUM LONGUS MUSCles AFTER FREE GRAFTING}

Bruce M. Carlson, MD, PhD, Kenneth R. Wagner, PhD, and Stephen R. Max, PhD

Abstract: Free grafts of the extensor digitorum longus (EDL) muscle of the rat are invaded by regenerating nerve fibers during the second postoperative week. Neuromuscular junctions are established during the third week. Levels of choline acetyltransferase (CAT) activity parallel the invasion of the graft by nerve fibers, but this activity never returns to control levels. We conclude that incomplete reinnervation of free muscle grafts may be a factor in the failure of such grafts to reattain the mass of control muscles.

MUSCLE \& NERVE 2:304-307 1979

Autogenous free muscle grafts have proved useful in several respects in the study of skeletal muscle regeneration. ${ }^{3}$ The procedure is such that the grafted muscle is cut off from the beginning from both a neural and a vascular supply. Blood vessels must enter the graft before muscle regeneration can begin, and reinnervation must occur if the regenerative process is to be completed.

Results of a recent study, which examined the role of enzymes associated with glucose metabolism in regenerating free grafts of the extensor digitorum longus (EDL) muscle, were consistent with the interpretation that the grafts did not attain complete maturity, at least not in the biochemical sense. ${ }^{13}$ It is possible that the lack of biochemical maturation, as well as the failure of free muscle grafts to attain the mass of a normal muscle, might reflect deficient innervation of the grafts. Indeed, Hall-Craggs and Brand ${ }^{7}$ showed that facilitation of reinnervation at the time of grafting markedly enhances the extent of regeneration.

We conducted a correlative morphologic and biochemical study to determine (1) the relationship between early reinnervation of the graft and choline acetyltransferase (CAT) activity; and (2) the extent to which the innervation of a mature free muscle graft approaches that of the normal ungrafted muscle. These data have appeared elsewhere in preliminary form. ${ }^{14}$

\section{MATERIALS AND METHODS}

All operations were conducted on male SpragueDawley rats (Charles River) weighing 175-200 g. During surgical procedures, the rats were anesthetized with ether. Upon removal, the right EDL muscle was injected with $100-200 \mu$ of bupivacaine (Marcaine) solution $0.75 \%$ bupivacaine in $0.9 \% \mathrm{NaCl}$, Winthrop Laboratories, New York, NY). The muscle was then soaked in bupivacaine solution for $10 \mathrm{~min}$ to expose the peripheral fibers to the effects of the local anesthetic. The muscle was immediately grafted back into its own bed and sutured to its proximal and distal tendons. No attempt was made to reestablish nervous or vascular continuity between graft and host. The left EDL remained untouched and served as a normal con-

From the Department of Anatomy, University of Michigan School of Medicine, Ann Arbor, MI (Dr. Carlson); and the Departments of Neurology and Pediatrics, University of Maryland School of Medicine, Baltimore, MD (Drs, Max and Wagner)

Acknowledgments: This work was supported by grants from the Muscular Dystrophy Association of America, the National Amyotrophic Lateral Sclerosis Foundation, and $\mathrm{NIH}$ (NS-13116).

Address reprint requests to Dr. Carison at the Department of Anatomy, 4622 Medical Sciences II, University of Michigan, Ann Arbor, MI 48109.

Received for publication September 13,1978 ; revised manuscript accepted for publication December 28, 1978.

0148-639x/0204/0304 $\$ 00.00 / 0$

1979 Houghton Mifflin Professional Publishers 


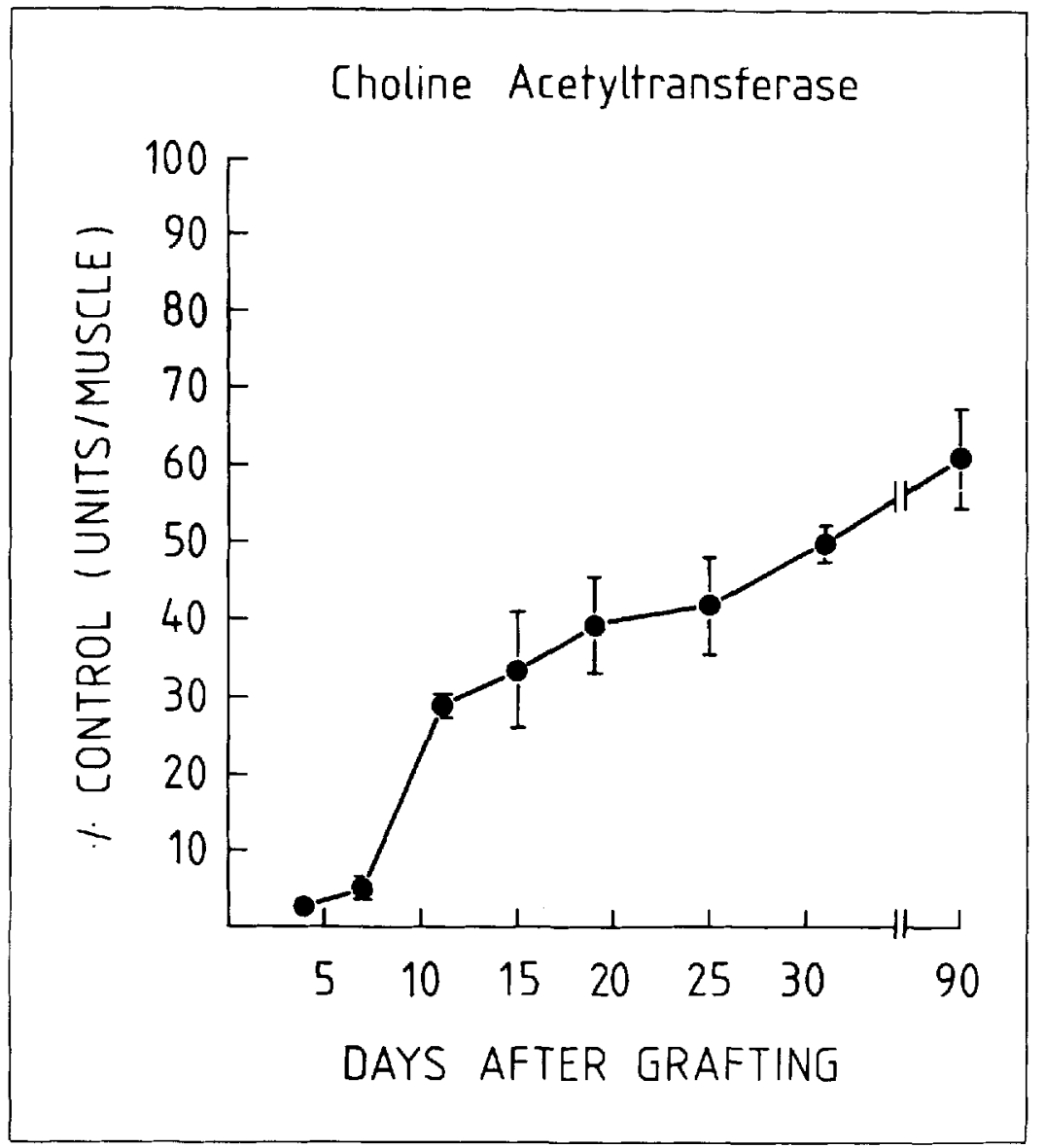

Figure 1. Graph showing choline acetyltransferase activity of freely grafted EDL muscles of the rat at various periods after transplantation. The data are expressed as percentage of activity in contralateral control muscles (units per muscle). trol. At 4, 7, 11, 15, 19,25, 31, and 90 days postoperatively, both the graft and its contralateral counterpart were removed from each of six to seven rats. The muscles were weighed and immediately immersed in dry ice and isopentane, after which they were kept continuously frozen on dry ice during the two-day shipping period from Michigan to Maryland. Upon arrival, muscles were minced with scissors in ice-cold $0.01 \mathrm{M}$ sodium phosphate buffer, $\mathrm{pH} 7.4$, and were homogenized by hand using a Tenbroeck homogenizer (Kontes Glass Co., Vineland, NJ). Homogenates were used for analysis of CAT.

Morphologic Analysis. Grafted muscles from a parallel series of rats were examined histologically at each of the times when muscles were removed for enzyme assay, as well as at daily intervals up to 20 days. These grafts were fixed in Bouin's solution; cross and longitudinal sections were stained with hematoxylin and eosin (H \& E) or with Palmgren's silver technique ${ }^{11}$ for the staining of nerve fibers.

Other grafts were frozen in dry ice and isopentane, after which they were sectioned with a cryo- stat. Sections were stained by the Gomori method for cholinesterase activity ${ }^{12}$ to demonstrate motor endplates, or by Goshgarian's technique, ${ }^{6}$ which combines a silver stain for nerve fibers with histochemical staining for motor endplates.

Cat Assay. CAT, an enzyme that serves as a biochemical marker for the formation of cholinergic synapses, ${ }^{4}$ was assayed as described by Fonnun ${ }^{5}$ and modified for choline specificity. ${ }^{9}$ Because of the characteristic fluctuations in the weights of the muscle grafts during this period, ${ }^{3}$ it was decided that CAT activity per muscle, rather than specific activity, provided the most accurate means of expressing the data. The mean CAT activity of 45 control muscles was $14.39+1.11 \mathrm{nmole} / \mathrm{hr} /$ muscle.

\section{RESULTS}

CAT was assayed as a marker for reinnervation. By day 4, after autogenous free grafting of Marcaine-treated muscles, CAT had decreased to $2 \%$ of control activity (fig. 1). By day 7 , activity was still minimal, at $7 \%$ of control. Between days 7 and 11 , there was a fourfold increase in activity, to $27 \%$ of 


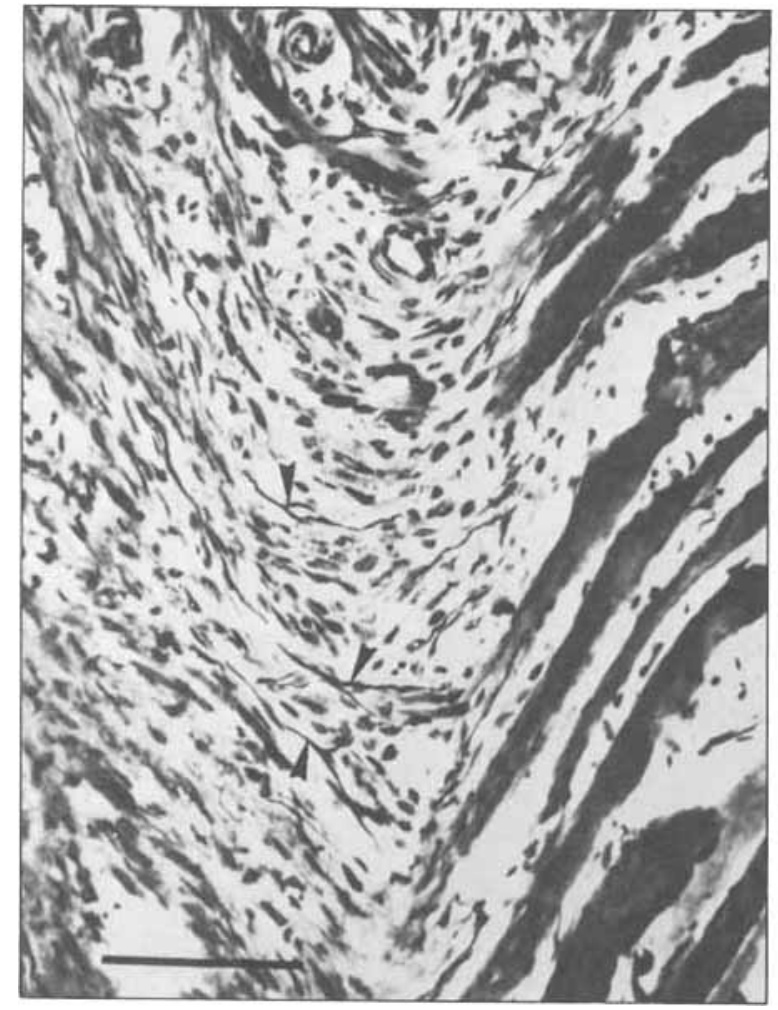

Figure 2. Photomicrograph showing the ingrowth of regenerating nerve fibers (arrows) into a 10-day free graft of the EDL muscie in the rat. Palmgren's silver stain. Bar $=100 \mu \mathrm{m}$

control, after which enzyme activity rose steadily to $60 \%$ of control by day 90 . The overall increase in CAT during the 90-day period was about 30 fold (fig. 1). Nevertheless, only $60 \%$ of control activity was attained, indicating incomplete reinnervation of the grafted muscles. The fourfold in. crease in CAT between 7- and 11-day muscle grafts should be compared with the 200 -fold increase observed during the formation of neuromuscular junctions in the developing chick iris. ${ }^{4}$

The changes in CAT activity correlated well with morphologic observations. During the first week after grafting, only degenerating nerve fibers were present within the grafts. Nerve fibers began penetrating the proximal regions of the grafts during the second week (fig. 2) and were present in substantial numbers by day 11 . Within a few days of their penetration into the grafts, the regenerating nerve fibers became closely associated with muscle fibers. Motor end plates became established during the third week (fig. 3), and reinnervation was seen in some regenerated muscle spindles. After the third week, no major qualitative changes in the innervation of the grafts were evident.

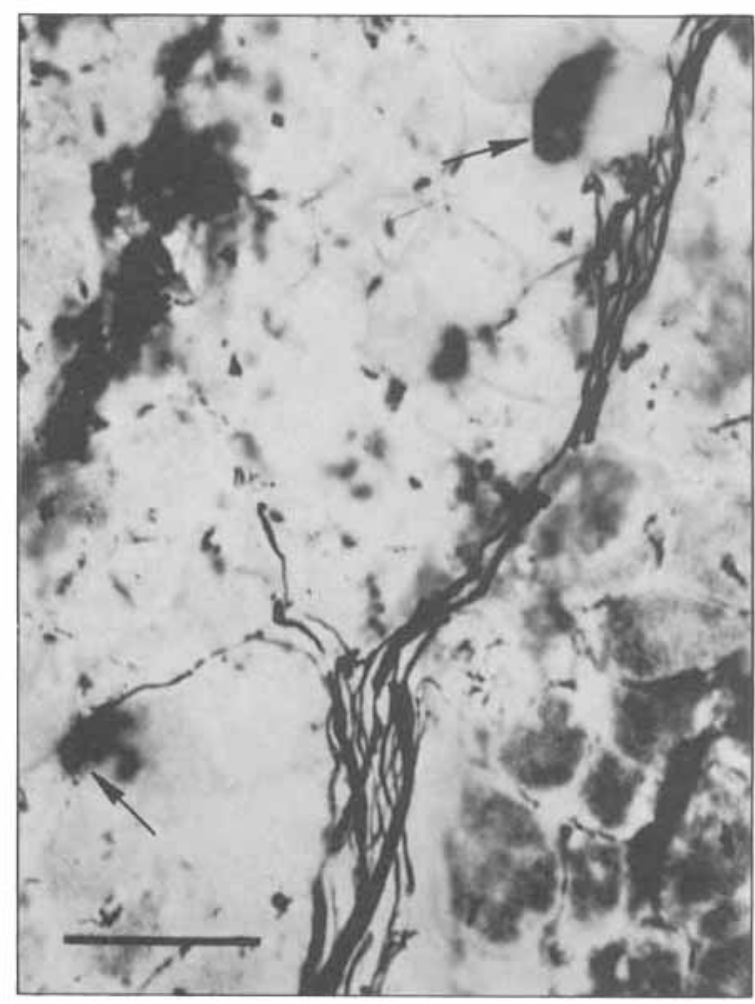

Figure 3. Photomicrograph showing the comomed staining of nerve fibers and motor endplates (arrows) in a thick section of a 23-day graft of the EDL muscle in the rat. Goshgarian's combined stain. Bar $=50 \mu \mathrm{m}$.

\section{DISCussion}

It is very difficult to estimate the extent of innervation of a muscle graft by morphologic methods alone. The CAT data provide a more readily interpretable quantitative picture of the nerve supply. The sharp rise in CAT activity paralleled the morphologic evidence of nerve ingrowth into the grafts during this period.

In mature grafts, the CAT data show consistently lower levels of innervation on a whole-muscle basis in grafts than in the normal control muscles. These lower levels are correlated with the lower-than-normal weights of the grafts. It is noteworthy that despite the lower weights, mature (60day) muscle grafts contain normal numbers of muscle fibers. ${ }^{2}$ In fact, ongoing studies ${ }^{3}$ have shown that at least some grafts in the 15- to 20-day range possess somewhat more than the normal number of muscle fibers. This is probably due either to regeneration of more than one muscle fiber within the basement membranes of degenerated muscle fibers or, possibly, to interstitial regeneration of new muscle fibers. ${ }^{8}$ Extreme atrophy of isolated regenerated muscle fibers occurs during the course 
of development of EDL muscle grafts, but this atrophy is not extensive cnough to account for the small masses of mature grafts. These are probably attributable to the lack of attainment of normal cross-sectional areas of the regenerating muscle fibers. In completely denervated muscle regenerates, Mong ${ }^{10}$ noted gradual atrophy of the muscle fibers after 30 days. Another possible correlate with a reduced innervation of the grafts is the presence of significant numbers of myo-myous junctions between regenerating muscle fibers. ${ }^{8}$ The function of these structures is not well known, but they may represent compensatory adaptations for communication among sparsely innervated muscle fibers.

The reason for the quantitatively deficient innervation of muscle grafts is not known. Death of damaged neurons, insufficient penetration of the graft, and reduced branching of nerve fibers within the grafts are all possibilities, but there is not sufficient experimental information to resolve the question at this time. Deficient reinnervation could account for the poor recovery of muscle weight, the reduced activity of a number of enzymes, ${ }^{13}$ and the persistence of abnormal morphologic characteristics-e.g., the persistence of central nuclei.

Histochemically stainable acetylcholinesterase (AChE) motor endplates persist throughout the postoperative period. Because of this, it is difficult to determine whether stained motor endplates are old or new, unless the stain is combined with a technique demonstrating nerve axons leading to the endplates. Bennett et $a l^{1}$ in the minced soleus, and Hansen-Smith and Carlson in EDL grafts, have demonstrated the staining of motor endplates for two to three weeks after the initial operation in legs that had been denervated. Thus, old motor endplates retain AChE activity for at least this period. Total AChE and AChE specific activity (data not shown) changed very little over the experimental period. This is a reflection of the overlap between the decline of old motor endplates and the formation of new ones.

We conclude that incomplete reinnervation may be a factor contributing to the less-than-normal functional recovery of grafted muscles, and that efforts should be directed toward the establishment of complete reinnervation.

\section{REFERENCES}

1. Bennett MR, Florin T, Woog R: The formation of synapses in regenerating mammalian striated muscle. $J$ Physiol (Lond) 238:79-92, 1974 .

2. Carlson BM: A quantitative study of muscle fiber survival and regeneration in normal, predenervated and Marcainetreated free muscle grafts in the rat. Exp Neurol 52:421432, 1976 .

3. Carlson BM: A review of muscle transplantation in mammals. Physiol Bohemoslov, 27:387-400, 1979.

4. Chiapenelli V, Giacobine E, Pilar G, Uchimura H: Induction of cholinergic enzymes in chick ciliary ganglion and iris muscle cells during synapse formation.J Physiol (Lond) $257: 749-766,1975$.

5. Fonnun F: A rapid radiochemical method for the determination of choline acetyltransferase. J Neurochem 24:407$409,1975$.

6. Goshgarian HG: A rapid silver impregnation for central and peripheral nerve fibers in paraf fin and frozen sections. Exp Neurol 57:296-301, 1977 .

7. Hall-Craggs ECB, Brand P: Effects of previous nerve injury on the regeneration of free autogenous muscle grafts.
Exp Neurol 57:275-281, 1977.

8. Hansen-Smith FM, Carlson BM: Cellular responses to free grafting of the extensor digitorum longus muscle of the rat. $J$ Neurol Sci, in press.

9. Max SR, Rifenberick DH: Choline acetyltransferase and acetylcholinesterase in skeletal muscle regeneration. J Neurochem 24:771-773, 1975.

10. Mong FSF: Histological and histochemical studies on the nervous influence on minced muscle regeneration of triceps surae of the rat. $J$ Morphol 151:451-462, 1977.

11. Palmgren A: Specific silver staining of nerve fibers. I. Techniques for vertebrates. Acta Zool 41:239-265, 1960.

12. Pearse AGE: Histochemistry, vol 2, 3rd ed. London, Churchill Livingstone, 1972, p 1312.

13. Wagner KR, Carlson BM, Max SR: Developmental patterns of glycolytic enzymes in regenerating skeletal muscle after autogenous free grafting. J Neurol Sci 34:373-390, 1977.

14. Wagner KR, Carlson BM, Max SR: Reinnervation of rat extensor digitorum longus muscles after autogenous free grafting. Neurosci Abs 4:535, 1978. 